\title{
A COMPARATIVE STUDY ON EMPLOYEE INTER PERSONAL AND HARMONIOUS RELATIONSHIPS WITH REFERENCE TO HPCL AND CFL
}

\author{
${ }^{1}$ Dr. Shaik Shamshuddin \\ ${ }^{1}$ Assistant Professor, \\ GITAM Institute of Management, \\ GITAM Deemed to be University.
}

\author{
${ }^{2}$ Dr. Shaik Khadar Baba \\ ${ }^{2}$ PA to Principal, \\ Department of Arts and Commerce, \\ Andhra University,
}

\author{
${ }^{3}$ Dr. Shaik Haniefuddin \\ ${ }^{3}$ Professor in Management, \\ SIMS, Visakhapatnam
}

\begin{abstract}
Research activity in the area of HRM/HRD is increasing and still there is a lot of scope for research to study the practices and their impact. The researcher has made an effort to review the studies related to the topic chosen. The studies reveal that there are a number of studies at macro level and that to them are more foreign studies which have given the basic knowledge to proceed. There are also some Indian Studies related to the present topic. There are hardly any studies related to Petrochemicals and Fertilizer Industries at the Micro Level. In this background, the main objective of the study is to assess the levels of the utilization of human resources as well as to determine the functional status of the units. Keeping the above aspects under consideration, the study selected two large scale industrial units Viz., The very purpose of this research is to know the opinion of the Employees on interpersonal relations among them for better implementation of HR Practices in Petrochemical and Fertilizer industries the effect of the development in industrial organizations and the development of productivity culture in the employees. The study demands direct answer from the respondents, which without any ambiguity will determine the factors which contribute positively or negatively for assessing the functioning and the effect of the HRD among selected industries.
\end{abstract}

\section{INTRODUCTION}

Human Resource Development is observed as a multi-disciplinary concept. Economists describe it from the economic angles of capital asset, labour, skill and wages. Further, they observed human resources as accumulation of capital and their effective investment for the future harvest of any nation for global development scenario. A psychologist considers HRD from the psychological dimensions of attitudes, aptitude values, intelligence, perceptions, aspirations and motivations, etc. Moreover, Psychology provides an explanation for a variety of human behaviour and also several remedial functions (through guidance, counselling etc.,). Similarly, a sociologists' perception of HRD moves around social relations, ingredients like family, groups, crowds, mob etc., while an Anthropologist looks at HRD keeping in view the history of humanity and focus as on several aspects of tradition, kinship, culture, myths and ceremonies.

The political scientists explain HRD in the light of different political ideologies which govern different societies. The discipline of management, which is the latest addition to the social sciences, explains HRD from the behavioural view point of science.

\section{NEED FOR THE STUDY}

It was observed that among all resources, human resource is the vital resource in deciding the success or failure of any organization and its sustainability. Further, the changes taking place in the technology transfer in production, online business strategies, competition among organisations, globalisation, etc. put tremendous pressure on human resources in the existing and future scenario. Hence, 
the prime requirement is not only to enrich human resources but also to utilize human resources effectively to face existing and future challenges in short and long term perspectives. Keeping the above aspects under consideration, the study assessed the levels of the utilization in human resources in the varied conditions particularly in the Petro-Chemical and Fertilizer industries which have become core industrial category in the existing and future scenario. The Human Resources Development Policies of HPCL/CFL are not only fair and just to ensure that responsibility for results is provided right from the lower level so that employees can develop a sense of independence and self-confidence.

\section{SCOPE OF THE STUDY}

Research activity in the area of HRM/HRD is increasing and still there is a lot of scope for research to study the practices and their impact. The researcher has made an effort to review the studies related to the topic chosen. The studies reveal that there are a number of studies at macro level and that to them are more foreign studies which have given the basic knowledge to proceed. There are also some Indian Studies related to the present topic. There are hardly any studies related to Petrochemicals and Fertilizer Industries at the Micro Level. In this background, the researcher decided to pursue his studies at are micro level to study the HRD policies, practices and their impact by studying the perceptions of the selected employees in the two selected organizations. A comparative analysis was also made between the two organizations with reference to the HRD Practices. The required data was subject to statistical treatment by using the critical Ratio Method for establishing the validity and reliability of the instrument. It is proved after proper calculations that at 0.01 and 0.05 levels of significance all the statements were accepted. After eliminating the rejected statements at the level of significance, the final questionnaire with statements was approved for administration.

\section{OBJECTIVES OF THE STUDY}

- To assess the interpersonal relations between employees and Management in Selected Units.

- To assess the status of working conditions of the employees in the selected units.

- To trace the problems, bottlenecks and deficiencies arising in various spheres of Interpersonal Relations.
- To suggest feasible and amicable strategies for the optimum utilization of human resources and also for the up-gradation of the skills of human resources to face the challenges emerging in the existing and future scenario for better interpersonal relations and coordination.

\section{RESEARCH METHODOLOGY}

Before taking the initiatives of administering the questionnaire a pilot study was conducted in order to test the validity and appropriateness of the statements. Item validity of employees' questionnaire is determined by critical ratio values, comparing the upper $27 \%$ and lower $27 \%$ groups. Primary data was entered using SPSS (Statistical Package for Social Sciences) software and STATISTICA. Uni-variate and bi-variate tables were generated and chi-square tests and t-tests were carried out for testing the hypothesis at problem the level itself.

\section{The $\chi^{2}$ test:}

The Chi-square $\left(\chi^{2}\right)$ test is one of the simplest and most widely used non-parametric tests in statistical work. The symbol $\chi^{2}$ is the Greek letter chi. the $\chi^{2}$ test was first used by Karl Pearson in the year 1900 . The quantity $\chi^{2}$ describes the magnitude of the discrepancy between theory and observation.

$$
\text { It is defined as: } \chi^{2}=\Sigma \frac{(\mathrm{O}-\mathrm{E})^{2}}{\mathrm{E}}
$$

Where $\mathrm{O}$ refers to the observed frequencies and $\mathrm{E}$ refers to expected frequencies.

\section{The t-test:}

The t-distribution is an important statistical tool as number of variables taken in HPCL/CFL. The following are some of them;

1. $\mathrm{t}$-test for significance for single means, population variables being unknown.

2. $\mathrm{t}$-test for the significance of the difference between two sample means, the population variance being equal but unknown.

3. t-test for significance of an observed sample correlation coefficient

$\mathrm{t}$-test was used for testing the significance of the difference between two sample means.

The formulae used for this test is 
EPRA International Journal of Multidisciplinary Research (IJMR) - Peer Reviewed Journal Volume: 6 | Issue: 1 | January 2020 || Journal DOI: 10.36713/epra2013 || SJIF Impact Factor: 5.614||ISI Value: 1.188

$$
\begin{aligned}
& \overline{\mathrm{X}}-\overline{\mathrm{Y}} \quad \sim \operatorname{tn}_{1}+\mathrm{n}_{2}-2 \\
& \mathrm{t}=\frac{\mathrm{X}-\mathrm{Y}}{\mathrm{SX} \sqrt{1}+\underline{1}} \\
& \begin{array}{ll}
\mathrm{n}_{1} & \mathrm{n}_{2}
\end{array} \\
& \mathrm{X}=\underline{1} \Sigma \mathrm{X}, \quad \overline{\mathrm{Y}}=\underline{1} \Sigma \overline{\mathrm{Y}} \\
& \mathrm{n}_{1} \quad \mathrm{n}_{2} \\
& \text { And } \mathrm{S}^{2}=\frac{}{\mathrm{n}_{1}+\mathrm{n}_{2}-2} \\
& \left.\left[\Sigma(\overline{\mathrm{X}}-\mathrm{X})^{2}+\Sigma \overline{(\mathrm{Y}}-\mathrm{Y}\right)^{2}\right]
\end{aligned}
$$

Sample of the Study: from HPCL and 200 employees from CFL. The

GENDER-WISE DISTRIBUTION OF SAMPLE EMPLOYEES FROM THE SELECTED INDUSTRIES
\begin{tabular}{|c|c|c|c|}
\hline Gender & Hindustan Petroleum Co. Ltd. & $\begin{array}{c}\text { Coromandel } \\
\text { Fertilizers }\end{array}$ & Total \\
\hline Male & $167(83.5)$ & $171(85.5)$ & $338(84.5)$ \\
\hline Female & $33(16.5)$ & $29(14.5)$ & $62(15.5)$ \\
\hline Total & $\mathbf{2 0 0 ( 1 0 0 . 0 )}$ & $\mathbf{2 0 0 ( 1 0 0 . 0 )}$ & $\mathbf{4 0 0 ( 1 0 0 . 0 )}$ \\
\hline
\end{tabular}

The table given above explains gender-wise distribution of sample employees from the two selected industries i.e. Hindustan Petroleum Corporation Limited (HPCL) and Coromandel Fertilizers Limited (CFL). Out of the selected employees from HPCL the majority $(83.5 \%)$ is male and the remaining 16.5 per cent are female. From the total sample from CFL the higher group $(85.5 \%)$ is male and few $(14.5 \%)$ are female employees.

AGE-WISE DISTRIBUTION OF SAMPLE EMPLOYEES FROM THE SELECTED INDUSTRIES

\begin{tabular}{|c|c|c|c|}
\hline Age & Hindustan Petroleum Co. Ltd. & $\begin{array}{c}\text { Coromandel } \\
\text { Fertilizers }\end{array}$ & Total \\
\hline Below 30 & $17(8.5)$ & $5(2.5)$ & $22(5.5)$ \\
\hline $30-40$ & $68(34.0)$ & $77(38.5)$ & $145(36.3)$ \\
\hline $41-50$ & $83(41.5)$ & $90(45.0)$ & $173(43.3)$ \\
\hline Above 50 & $32(16.0)$ & $28(14.0)$ & $60(15.0)$ \\
\hline Total & $\mathbf{2 0 0 ( 1 0 0 . 0 )}$ & $\mathbf{2 0 0 ( 1 0 0 . 0 )}$ & $\mathbf{4 0 0 ( 1 0 0 . 0 )}$ \\
\hline
\end{tabular}

Education wise Distribution

\begin{tabular}{|c|c|c|c|}
\hline Education & Hindustan Petroleum Co. Ltd. & $\begin{array}{c}\text { Coromandel } \\
\text { Fertilizers }\end{array}$ & Total \\
\hline Diploma & $37(18.5)$ & $67(33.5)$ & $104(26.0)$ \\
\hline Degree & $59(29.5)$ & $88(44.0)$ & $147(36.8)$ \\
\hline Engineering & $62(31.0)$ & $25(12.5)$ & $87(21.8)$ \\
\hline P.G. & $42(21.0)$ & $20(10.0)$ & $62(15.5)$ \\
\hline Total & $\mathbf{2 0 0 ( 1 0 0 . 0 )}$ & $\mathbf{2 0 0 ( 1 0 0 . 0 )}$ & $\mathbf{4 0 0 ( 1 0 0 . 0 )}$ \\
\hline
\end{tabular}


ISSN (Online): 2455-3662

EPRA International Journal of Multidisciplinary Research (IJMR) - Peer Reviewed Journal Volume: 6 | Issue: 1 | January 2020 || Journal DOI: 10.36713/epra2013 || SJIF Impact Factor: 5.614||ISI Value: 1.188

Designation wise Distribution

\begin{tabular}{|c|c|c|c|}
\hline Designation & $\begin{array}{c}\text { Hindustan Petroleum Co. } \\
\text { Ltd. }\end{array}$ & $\begin{array}{c}\text { Coromandel } \\
\text { Fertilizers }\end{array}$ & Total \\
\hline Worker & $63(31.5)$ & $61(30.5)$ & $124(31.0)$ \\
\hline Supervisor & $32(16.0)$ & $32(16.0)$ & $64(16.0)$ \\
\hline Clerk & $43(21.5)$ & $55(27.5)$ & $98(24.5)$ \\
\hline Engineer & $35(17.5)$ & $38(19.0)$ & $73(18.3)$ \\
\hline Officer & $27(13.5)$ & $14(7.0)$ & $41(10.3)$ \\
\hline Total & $\mathbf{2 0 0 ( 1 0 0 . 0 )}$ & $\mathbf{2 0 0 ( 1 0 0 . 0 )}$ & $\mathbf{4 0 0 ( 1 0 0 . 0 )}$ \\
\hline
\end{tabular}

Functional wise distribution

\begin{tabular}{|c|c|c|c|}
\hline Department & $\begin{array}{c}\text { Hindustan Petroleum Co. } \\
\text { Ltd. }\end{array}$ & $\begin{array}{c}\text { Coromandel } \\
\text { Fertilizers }\end{array}$ & Total \\
\hline Administrative & $33(16.5)$ & $26(13.0)$ & $59(14.8)$ \\
\hline Personal & $27(13.5)$ & $27(13.5)$ & $54(13.5)$ \\
\hline Finance & $23(11.5)$ & $20(10.0)$ & $43(10.8)$ \\
\hline Mechanical & $23(11.5)$ & $26(13.0)$ & $49(12.3)$ \\
\hline Material & $17(8.5)$ & $12(6.0)$ & $29(7.3)$ \\
\hline Production & $46(23.0)$ & $56(28.0)$ & $102(25.5)$ \\
\hline Civil & $17(8.5)$ & $24(12.0)$ & $41(10.3)$ \\
\hline Research \& Planning & $14(7.0)$ & $9(4.5)$ & $23(5.8)$ \\
\hline Total & $\mathbf{2 0 0 ( 1 0 0 . 0 )}$ & $\mathbf{2 0 0 ( 1 0 0 . 0 )}$ & $\mathbf{4 0 0}(\mathbf{1 0 0 . 0})$ \\
\hline
\end{tabular}

Experience wise Distribution

\begin{tabular}{|c|c|c|c|}
\hline Work Experience & $\begin{array}{c}\text { Hindustan Petroleum Co. } \\
\text { Ltd. }\end{array}$ & $\begin{array}{c}\text { Coromandel } \\
\text { Fertilizers }\end{array}$ & Total \\
\hline Below 5 & $10(5.0)$ & $9(4.5)$ & $19(4.8)$ \\
\hline $5-10$ & $74(37.0)$ & $65(32.5)$ & $139(34.8)$ \\
\hline $11-15$ & $92(46.0)$ & $98(49.0)$ & $190(47.5)$ \\
\hline Above 15 & $24(12.0)$ & $28(14.0)$ & $52(13.0)$ \\
\hline Total & $\mathbf{2 0 0 ( 1 0 0 . 0 )}$ & $\mathbf{2 0 0 ( 1 0 0 . 0 )}$ & $\mathbf{4 0 0 ( 1 0 0 . 0 )}$ \\
\hline
\end{tabular}

Team Work and Coordination

\begin{tabular}{|c|c|c|c|c|c|c|c|}
\hline Industry & $\begin{array}{c}\text { Not at all } \\
\text { true }\end{array}$ & $\begin{array}{c}\text { Rarely } \\
\text { True }\end{array}$ & $\begin{array}{c}\text { Sometimes } \\
\text { True }\end{array}$ & $\begin{array}{c}\text { Mostly } \\
\text { True }\end{array}$ & $\begin{array}{c}\text { Almost } \\
\text { always True }\end{array}$ & $\begin{array}{c}\text { Total } \\
\text { Chi-square } \\
\text { value }\end{array}$ \\
\hline Hindustan Petroleum Co. & 10 & 17 & 82 & 80 & 11 & 200 & \\
Ltd. & $(5.0)$ & $(8.5)$ & $(41.0)$ & $(40.0)$ & $(5.5)$ & $(100.0)$ \\
\hline Coromandel Fertilizers & 8 & 43 & 90 & 47 & 12 & 200 & $20.48^{* *}$ \\
$(4.0)$ & $(21.5)$ & $(45.0)$ & $(23.5)$ & $(6.0)$ & $(100.0)$ \\
\hline Total & $\mathbf{1 8}$ & $\mathbf{6 0}$ & $\mathbf{1 7 2}$ & $\mathbf{1 2 7}$ & $\mathbf{2 3}$ & $\mathbf{4 0 0}$ \\
$(\mathbf{1 0 0 . 0 )}$ & \\
\hline
\end{tabular}

The calculated chi-square value of 20.48 is significant at 0.01 level. 
EPRA International Journal of Multidisciplinary Research (IJMR) - Peer Reviewed Journal Volume: 6 | Issue: 1 | January 2020 || Journal DOI: 10.36713/epra2013 || SJIF Impact Factor: 5.614||ISI Value: 1.188

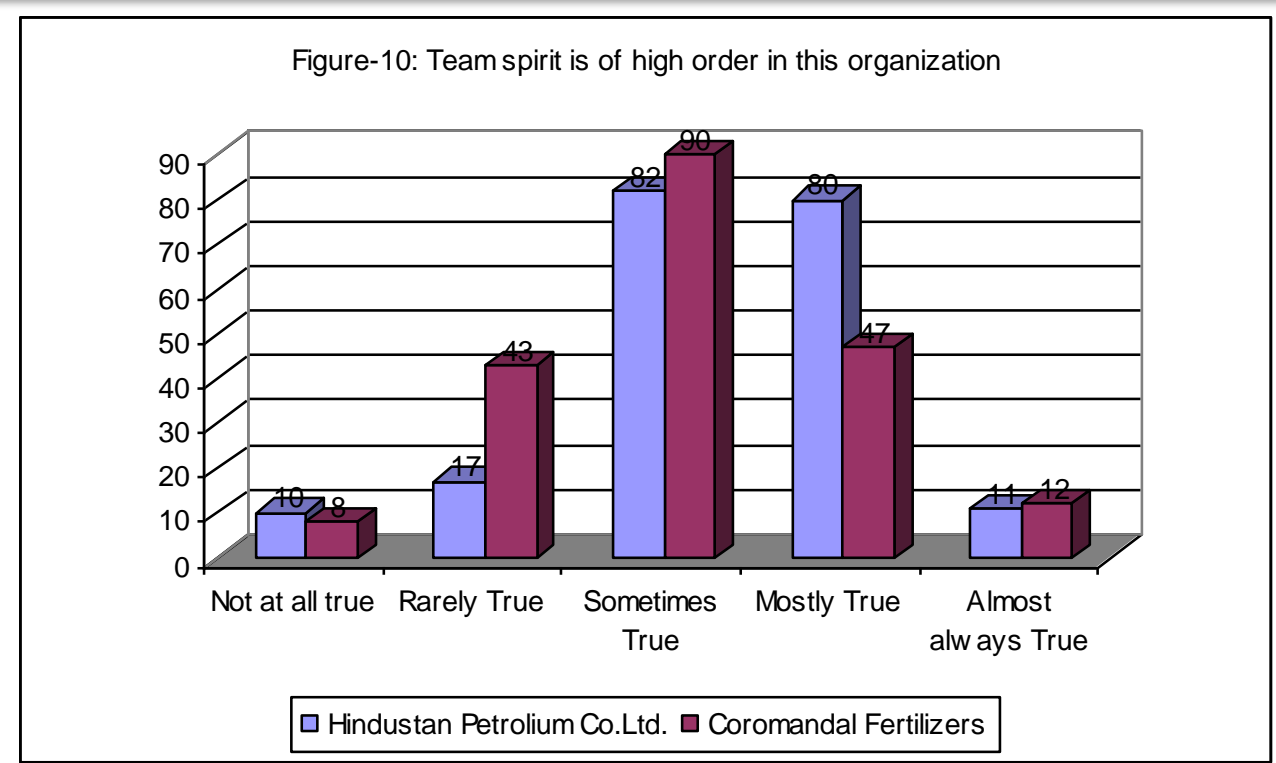

\section{Coordination in Problem Solving}

\begin{tabular}{|c|c|c|c|c|c|c|c|}
\hline Industry & $\begin{array}{c}\text { Not at } \\
\text { all true }\end{array}$ & $\begin{array}{c}\text { Rarely } \\
\text { True }\end{array}$ & $\begin{array}{c}\text { Sometimes } \\
\text { True }\end{array}$ & $\begin{array}{c}\text { Mostly } \\
\text { True }\end{array}$ & $\begin{array}{c}\text { Almost } \\
\text { always True }\end{array}$ & $\begin{array}{l}\text { Total } \\
\text { square } \\
\text { value }\end{array}$ \\
\hline $\begin{array}{c}\text { Hindustan Petroleum Co. } \\
\text { Ltd. }\end{array}$ & $\begin{array}{c}9 \\
(4.5)\end{array}$ & $\begin{array}{c}42 \\
(21.0)\end{array}$ & $\begin{array}{c}70 \\
(35.0)\end{array}$ & $\begin{array}{c}65 \\
(32.5)\end{array}$ & $\begin{array}{c}14 \\
(7.0)\end{array}$ & $\begin{array}{c}200 \\
(100.0)\end{array}$ \\
\hline Coromandel Fertilizers & 22 & 36 & 42 & 77 & 23 & 200 & $(11.5)$ \\
$(11.0)$ & $(18.0)$ & $(21.0)$ & $(38.5)$ & $(100.0)$ & $16.12^{* *}$ \\
& & $\mathbf{3 1}$ & $\mathbf{7 8}$ & $\mathbf{1 1 2}$ & $\mathbf{1 4 2}$ & $\mathbf{3 7}$ & $\mathbf{4 0 0}$ \\
$\mathbf{( 1 9 . 5 )}$ & $\mathbf{( 2 8 . 0 )}$ & $\mathbf{( 3 5 . 5 )}$ & $\mathbf{( 9 . 3 )}$ & $\mathbf{( 1 0 0 . 0 )}$ & \\
\hline
\end{tabular}

The generated chi-square value is 16.12 is significant at 0.01 level.

Figure-15: When problems arise people discuss these problems openly and try to solve them rather than keep accusing each other behind the back

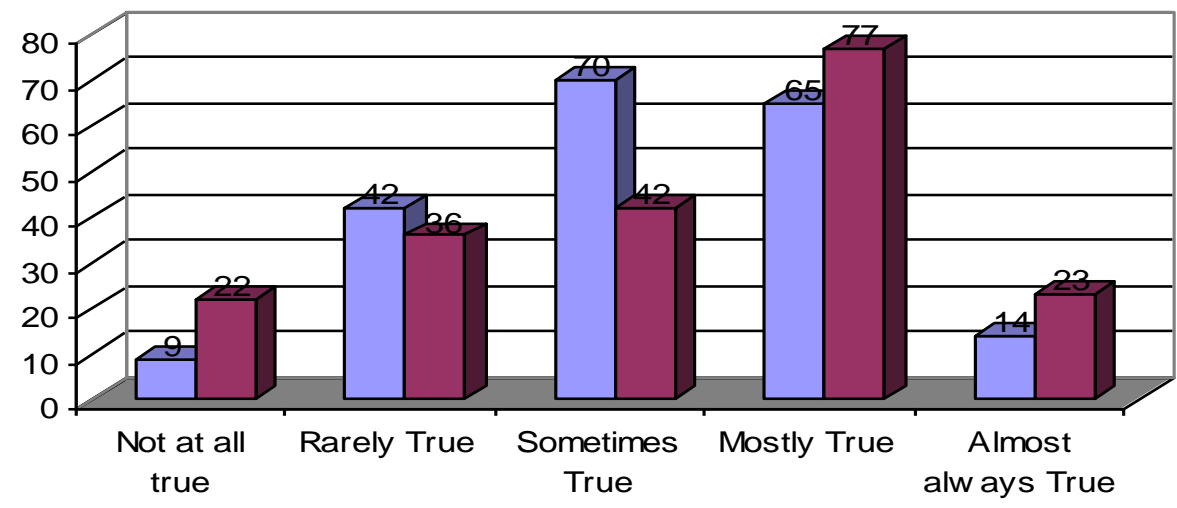

$\square$ Hindustan Petrolium Co.Ltd. $\square$ Coromandal Fertilizers 
ISSN (Online): 2455-3662

EPRA International Journal of Multidisciplinary Research (IJMR) - Peer Reviewed Journal Volume: 6 | Issue: 1 | January 2020 || Journal DOI: 10.36713/epra2013 || SJIF Impact Factor: 5.614||ISI Value: 1.188

Inter personal relations among employees

\begin{tabular}{|c|c|c|c|c|c|c|c|}
\hline Industry & $\begin{array}{c}\text { Not at all } \\
\text { true }\end{array}$ & $\begin{array}{c}\text { Rarely } \\
\text { True }\end{array}$ & $\begin{array}{c}\text { Sometimes } \\
\text { True }\end{array}$ & $\begin{array}{c}\text { Mostly } \\
\text { True }\end{array}$ & $\begin{array}{c}\text { Almost } \\
\text { always True }\end{array}$ & $\begin{array}{c}\text { Total } \\
\text { Chi-square } \\
\text { value }\end{array}$ \\
\hline Hindustan Petroleum Co. Ltd. & 3 & 45 & 70 & 47 & 35 & 200 & \\
$(1.5)$ & $(22.5)$ & $(35.0)$ & $(23.5)$ & $(17.5)$ & $(100.0)$ \\
\hline Coromandel Fertilizers & 3 & 24 & 78 & 74 & 21 & 200 \\
$(1.5)$ & $(12.0)$ & $(39.0)$ & $(37.0)$ & $(10.5)$ & $(100.0)$ & $16.35^{* *}$ \\
Total & $\mathbf{6}$ & $\mathbf{6 9}$ & $\mathbf{1 4 8}$ & $\mathbf{1 2 1}$ & $\mathbf{5 6}$ & $\mathbf{4 0 0}$ \\
$(\mathbf{1 0 0}$ & $\mathbf{( 1 4 . 0 )}$ & $(\mathbf{1 0 0 . 0})$ & \\
\hline
\end{tabular}

Figure-3.2 : Inter personal relations among employees

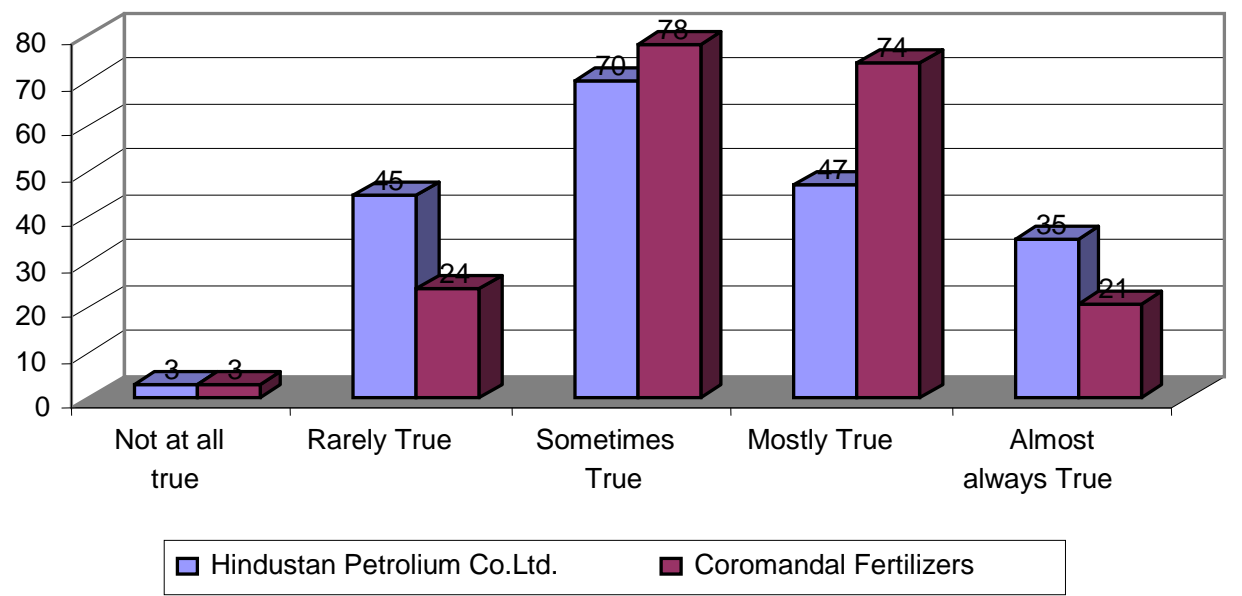

The generated chi-square value is 16.35 is significant at 0.01 level.

Cordial and Harmonious Relations

\begin{tabular}{|c|c|c|c|c|c|c|c|}
\hline Industry & $\begin{array}{c}\text { Not at all } \\
\text { true }\end{array}$ & $\begin{array}{c}\text { Rarely } \\
\text { True }\end{array}$ & $\begin{array}{c}\text { Sometimes } \\
\text { True }\end{array}$ & $\begin{array}{c}\text { Mostly } \\
\text { True }\end{array}$ & $\begin{array}{c}\text { Almost } \\
\text { always True }\end{array}$ & Total & $\begin{array}{c}\text { Chi-square } \\
\text { value }\end{array}$ \\
\hline $\begin{array}{c}\text { Hindustan Petroleum Co. } \\
\text { Ltd. }\end{array}$ & $\begin{array}{c}21 \\
(10.5)\end{array}$ & $\begin{array}{c}68 \\
(34.0)\end{array}$ & $\begin{array}{c}43 \\
(21.5)\end{array}$ & $\begin{array}{c}64 \\
(32.0)\end{array}$ & $\begin{array}{c}4 \\
(2.0)\end{array}$ & $\begin{array}{c}200 \\
(100.0)\end{array}$ & \\
\hline Coromandel Fertilizers & 5 & 47 & 66 & 76 & 6 & 200 & $19.96^{* *}$ \\
$(2.5)$ & $(23.5)$ & $(33.0)$ & $(38.0)$ & $(3.0)$ & $(100.0)$ & 1 \\
\hline Total & $\mathbf{2 6}$ & $\mathbf{1 1 5}$ & $\mathbf{1 0 9}$ & $\mathbf{1 4 0}$ & $\mathbf{1 0}$ & $\mathbf{4 0 0}$ \\
$\mathbf{( \mathbf { 1 0 . 5 }}$ & $\mathbf{( 2 8 . 8 )}$ & $\mathbf{( 2 7 . 3 )}$ & $\mathbf{( 3 5 . 0 )}$ & $\mathbf{( 2 . 5 )}$ & \\
\hline
\end{tabular}


EPRA International Journal of Multidisciplinary Research (IJMR) - Peer Reviewed Journal Volume: 6 | Issue: 1 | January 2020 || Journal DOI: 10.36713/epra2013 || SJIF Impact Factor: 5.614||ISI Value: 1.188

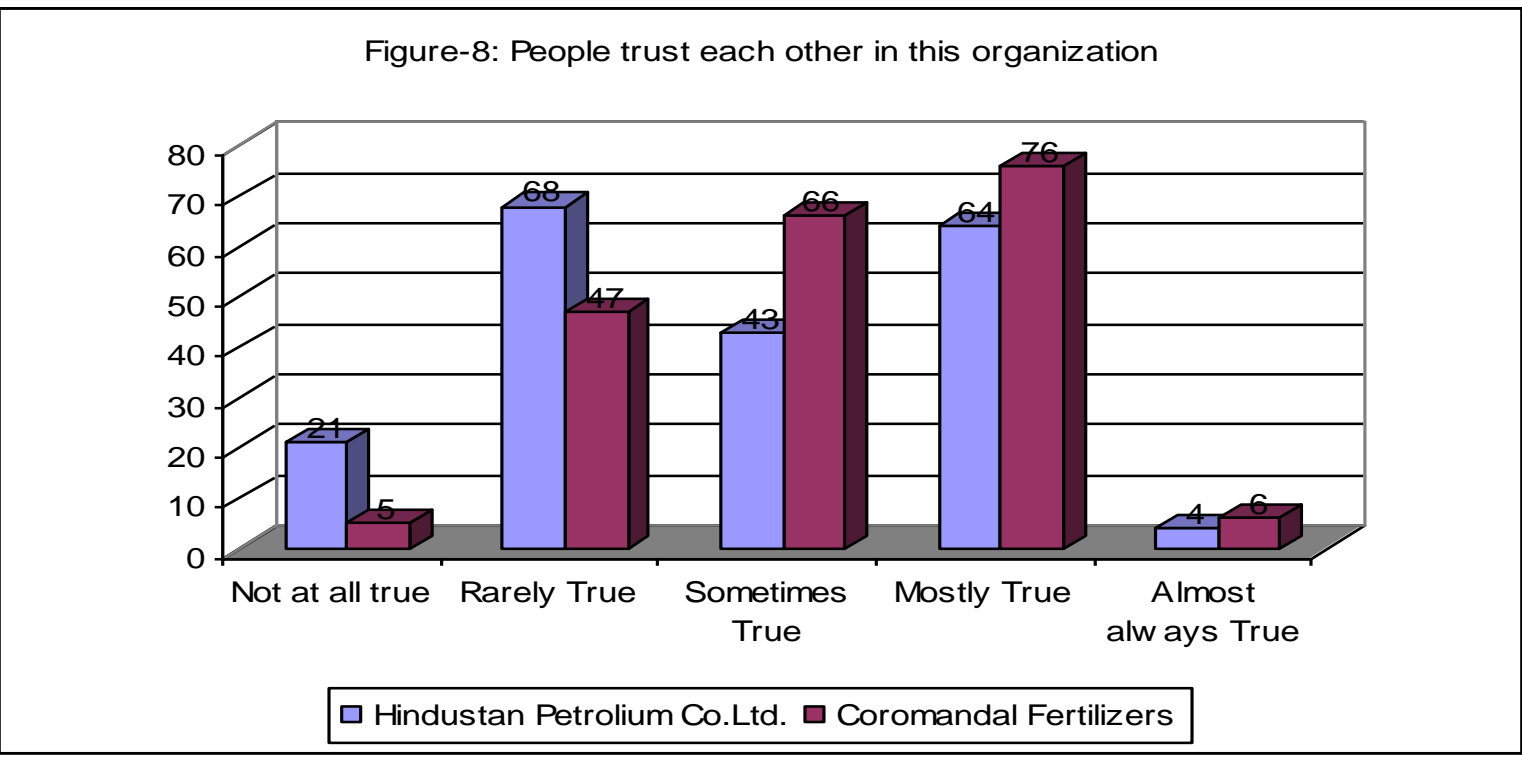

The generated chi-square value of 19.96 is not significant.

Delegating the Responsibility and coordination

\begin{tabular}{|c|c|c|c|c|c|c|c|}
\hline Industry & $\begin{array}{l}\text { Not at all } \\
\text { true }\end{array}$ & $\begin{array}{l}\text { Rarely } \\
\text { True }\end{array}$ & $\begin{array}{l}\text { Sometimes } \\
\text { True }\end{array}$ & $\begin{array}{l}\text { Mostly } \\
\text { True } \\
\end{array}$ & $\begin{array}{l}\text { Almost } \\
\text { always True }\end{array}$ & Total & $\begin{array}{l}\text { Chi-square } \\
\text { value }\end{array}$ \\
\hline Hindustan Petroleum Co. Ltd. & $\begin{array}{l}31 \\
(15.5)\end{array}$ & $\begin{array}{l}15 \\
(7.5)\end{array}$ & $\begin{array}{l}67 \\
(33.5)\end{array}$ & $\begin{array}{l}46 \\
(23.0)\end{array}$ & $\begin{array}{l}41 \\
(20.5)\end{array}$ & $\begin{array}{l}200 \\
(100.0)\end{array}$ & \multirow{3}{*}{8.66} \\
\hline Coromandel Fertilizers & $\begin{array}{l}39 \\
(19.5)\end{array}$ & $\begin{array}{l}24 \\
(12.0)\end{array}$ & $\begin{array}{l}77 \\
(38.5)\end{array}$ & $\begin{array}{l}31 \\
(15.5)\end{array}$ & $\begin{array}{l}29 \\
(14.5)\end{array}$ & $\begin{array}{l}200 \\
(100.0)\end{array}$ & \\
\hline Total & $\begin{array}{l}70 \\
(17.5)\end{array}$ & $\begin{array}{l}39 \\
(9.8)\end{array}$ & $\begin{array}{l}144 \\
(36.0)\end{array}$ & $\begin{array}{l}77 \\
(19.3)\end{array}$ & $\begin{array}{l}70 \\
(17.5)\end{array}$ & $\begin{array}{l}400 \\
(100.0)\end{array}$ & \\
\hline
\end{tabular}

The generated chi-square value of 8.66 is not significant.

Figure-25: Delegation of authority to encourage juniors to develop handling higher responsibilities is quite common in this organization

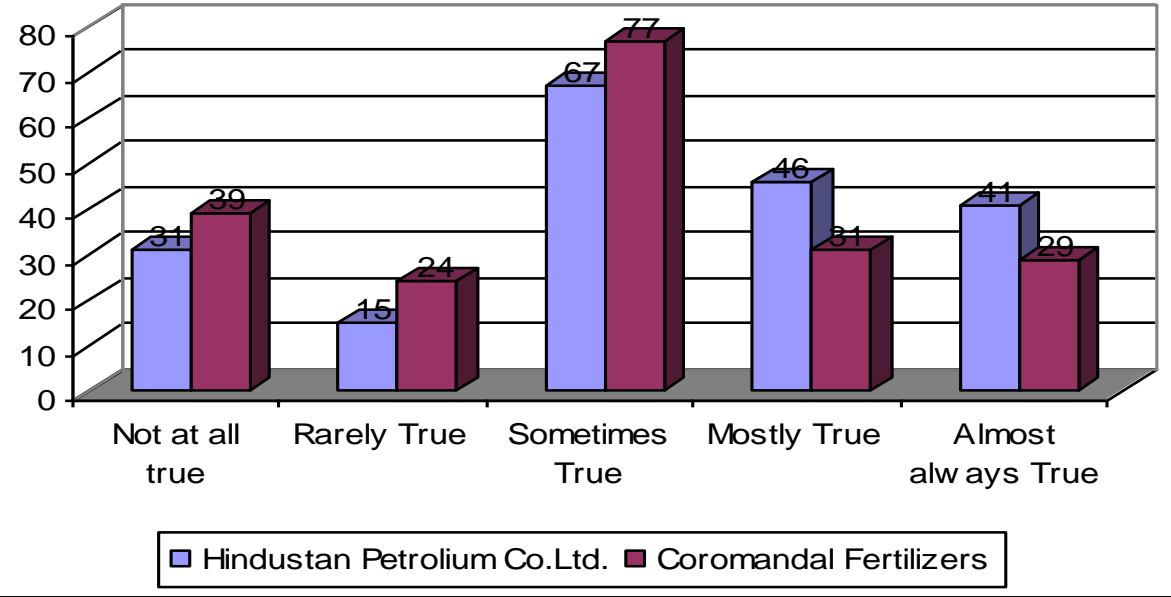


ISSN (Online): 2455-3662

EPRA International Journal of Multidisciplinary Research (IJMR) - Peer Reviewed Journal Volume: 6 | Issue: 1 | January 2020 || Journal DOI: 10.36713/epra2013 || SJIF Impact Factor: 5.614||ISI Value: 1.188

Quality Circles in helping environment

\begin{tabular}{|c|c|c|c|c|c|c|c|}
\hline Industry & $\begin{array}{c}\text { Not at all } \\
\text { true }\end{array}$ & $\begin{array}{c}\text { Rarely } \\
\text { True }\end{array}$ & $\begin{array}{c}\text { Sometimes } \\
\text { True }\end{array}$ & $\begin{array}{c}\text { Mostly } \\
\text { True }\end{array}$ & $\begin{array}{c}\text { Almost always } \\
\text { True }\end{array}$ & $\begin{array}{c}\text { Total } \\
\text { Chi-square } \\
\text { value }\end{array}$ \\
\hline Hindustan Petroleum Co. Ltd. & 4 & 5 & 58 & 95 & 38 & 200 & \\
$(2.0)$ & $(2.5)$ & $(29.0)$ & $(47.5)$ & $(19.0)$ & $(100.0)$ \\
\hline Coromandel Fertilizers & 3 & 18 & 65 & 94 & 20 & 200 \\
$(1.5)$ & $(9.0)$ & $(32.5)$ & $(47.0)$ & $(10.0)$ & $(100.0)$ \\
\hline Total & $\mathbf{7}$ & $\mathbf{2 3}$ & $\mathbf{1 2 3}$ & $\mathbf{1 8 9}$ & $\mathbf{5 8}$ & $\mathbf{4 0 0}$ \\
& $\mathbf{( 1 . 8 )}$ & $\mathbf{( 5 . 8 )}$ & $\mathbf{( 3 0 . 8 )}$ & $\mathbf{( 4 7 . 3 )}$ & $\mathbf{( 1 4 . 5 )}$ & $(\mathbf{1 0 0 . 0 )}$ & \\
\hline
\end{tabular}

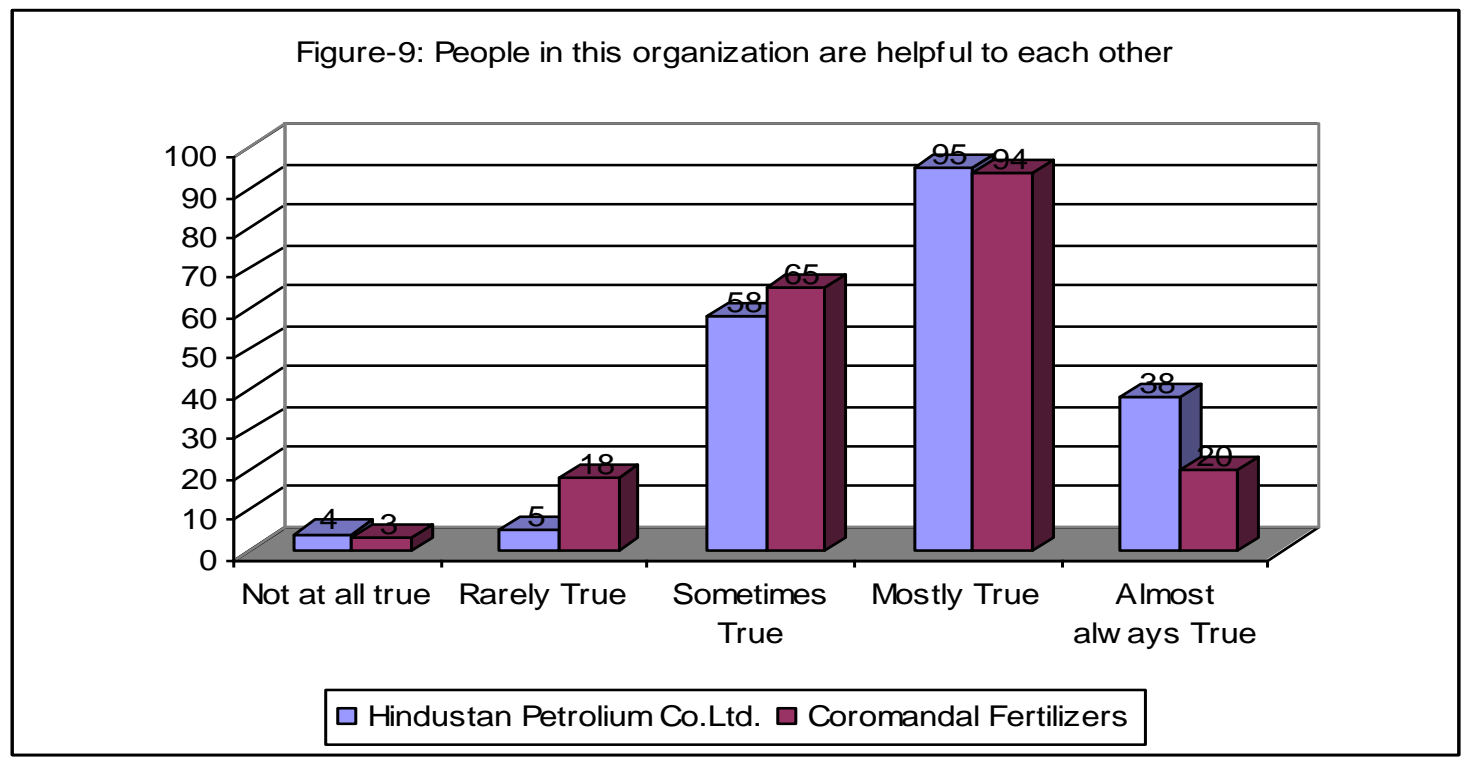

The generated chi-square value of 13.48 is significant at 0.01 level.

Status of Cooperation from Colleagues in Work Place

\begin{tabular}{|c|c|c|c|c|c|c|c|}
\hline Industry & $\begin{array}{c}\text { Not at all } \\
\text { true }\end{array}$ & $\begin{array}{l}\text { Rarely } \\
\text { True }\end{array}$ & $\begin{array}{c}\text { Sometimes } \\
\text { True }\end{array}$ & $\begin{array}{l}\text { Mostly } \\
\text { True }\end{array}$ & $\begin{array}{c}\text { Almost always } \\
\text { True }\end{array}$ & Total & $\begin{array}{c}\text { Chi- } \\
\text { square } \\
\text { value }\end{array}$ \\
\hline Hindustan Petroleum Co. Ltd. & $\begin{array}{c}23 \\
(11.5)\end{array}$ & $\begin{array}{c}58 \\
(29.0) \\
\end{array}$ & $\begin{array}{c}81 \\
(40.5)\end{array}$ & $\begin{array}{c}28 \\
(14.0)\end{array}$ & $\begin{array}{c}10 \\
(5.0)\end{array}$ & $\begin{array}{c}200 \\
(100.0)\end{array}$ & \multirow{3}{*}{$13.05^{*}$} \\
\hline Coromandel Fertilizers & $\begin{array}{c}36 \\
(18.0) \\
\end{array}$ & $\begin{array}{c}66 \\
(33.0) \\
\end{array}$ & $\begin{array}{c}49 \\
(24.5) \\
\end{array}$ & $\begin{array}{c}33 \\
(16.5) \\
\end{array}$ & $\begin{array}{c}16 \\
(8.0) \\
\end{array}$ & $\begin{array}{c}200 \\
(100.0)\end{array}$ & \\
\hline Total & $\begin{array}{c}59 \\
(14.8) \\
\end{array}$ & $\begin{array}{c}124 \\
(31.0) \\
\end{array}$ & $\begin{array}{c}130 \\
(32.5) \\
\end{array}$ & $\begin{array}{c}61 \\
(15.3) \\
\end{array}$ & $\begin{array}{c}26 \\
(6.5) \\
\end{array}$ & $\begin{array}{c}400 \\
(100.0)\end{array}$ & \\
\hline
\end{tabular}


EPRA International Journal of Multidisciplinary Research (IJMR) - Peer Reviewed Journal Volume: 6 | Issue: 1 | January 2020 || Journal DOI: 10.36713/epra2013 || SJIF Impact Factor: 5.614||ISI Value: 1.188

Figure-19: Status of cooperation from colleagues in w ork place is satisfactory

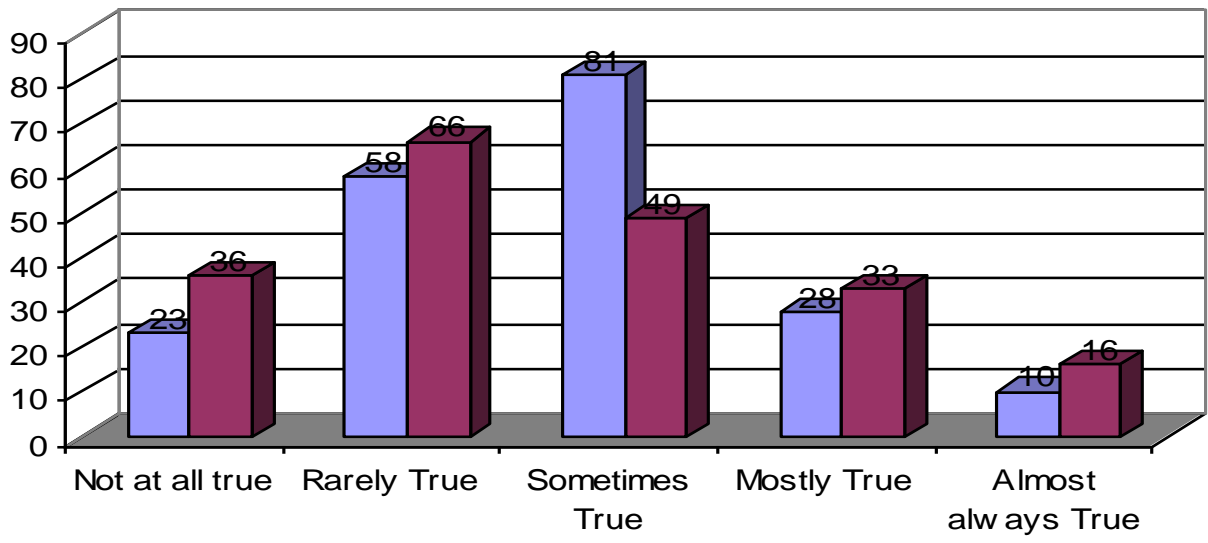

$\square$ Hindustan Petrolium Co.Ltd. $\square$ Coromandal Fertilizers

The generated chi-square value is 13.05 is significant at 0.05 level.

Active part of Senior Officers/Executives taking Interest in their juniors to help

\begin{tabular}{|c|c|c|c|c|c|c|c|}
\hline Industry & $\begin{array}{c}\text { Not at all } \\
\text { true }\end{array}$ & $\begin{array}{c}\text { Rarely } \\
\text { True }\end{array}$ & $\begin{array}{c}\text { Sometimes } \\
\text { True }\end{array}$ & $\begin{array}{c}\text { Mostly } \\
\text { True }\end{array}$ & $\begin{array}{c}\text { Almost } \\
\text { always True }\end{array}$ & $\begin{array}{l}\text { Total } \\
\text { square } \\
\text { value }\end{array}$ \\
\hline Hindustan Petroleum Co. Ltd. & 20 & 35 & 56 & 63 & 26 & 200 & \\
$(10.0)$ & $(17.5)$ & $(28.0)$ & $(31.5)$ & $(13.0)$ & $(100.0)$ \\
\hline Coromandel Fertilizers & 28 & 26 & 78 & 56 & 12 & 200 & $11.84^{*}$ \\
$(14.0)$ & $(13.0)$ & $(39.0)$ & $(28.0)$ & $(6.0)$ & $(100.0)$ \\
\hline Total & $\mathbf{4 8}$ & $\mathbf{6 1}$ & $\mathbf{1 3 4}$ & $\mathbf{1 1 9}$ & $\mathbf{3 8}$ & $\mathbf{4 0 0}$ \\
$\mathbf{( 1 2 . 0 )}$ & $\mathbf{( 1 5 . 3 )}$ & $\mathbf{( 3 3 . 5 )}$ & $\mathbf{( 2 9 . 8 )}$ & $\mathbf{( 9 . 5 )}$ & $(\mathbf{1 0 0 . 0 )}$ & \\
\hline
\end{tabular}

Figure-20: Senior officers /executives in this organization take active interest in their juniors and help them learn their job

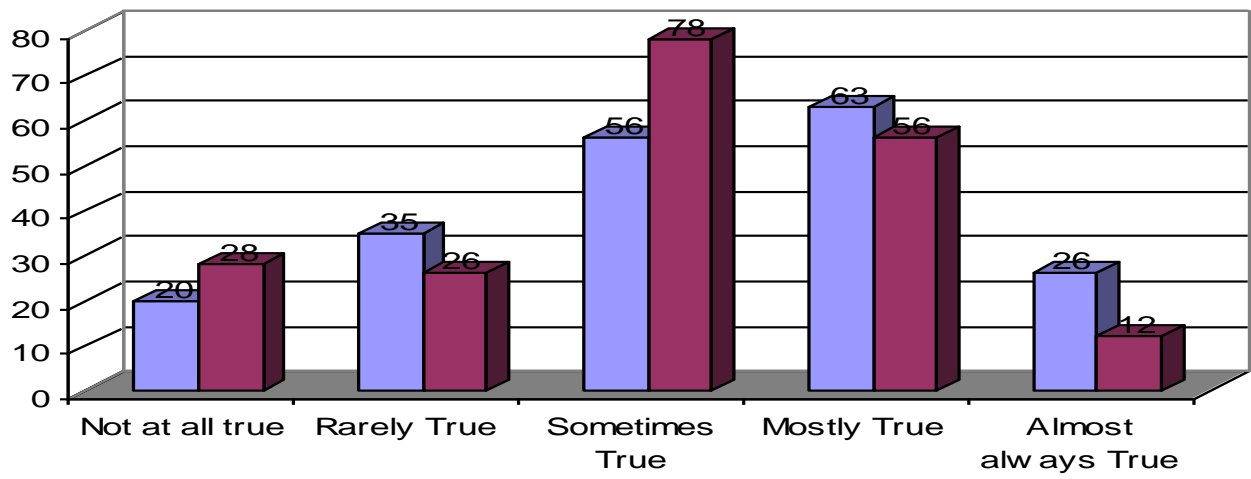

The calculated chi-square value of 11.84 is significant at 0.05 level.

FINDINGS AND OUTCOMES OF THE STUDY

1. There is no significant difference between the employees of the two industries on factors of Collaboration.

2. There is no significant difference between the employees of the two industries on factors of General Climate.

3. There is a significant difference in the opinion among the employees working in both the industries about employees not afraid to express or discuss their feelings with their superiors.

4. There is a significant difference between the employees from the above two industries that when any employee make a mistake, his/her supervisors treat that with understanding and help them to learn from such.

5. There is a significant difference in the opinion between the employees working 
in two industries that people trust each other in the organization.

6. Team spirit is of high order in this organization, people responding to this issue was expressed differently of various employees of two companies.

7. Employees felt that when seniors delegate authority to juniors, the juniors use it as an opportunity for development.

8. The status of cooperation from colleagues in work place is satisfactory, in both the organizations.

9. The HPCL employees found more significant on seniors' guidance to their juniors and prepare them for future responsibilities/ roles they are likely to take up than CFL employees.

\section{SUGGESTIONS}

- As it is observed communication plays a crucial role in improving the relationships among the employees who are not in a position to share their personal experiences with their colleagues and superiors.

- It is suggested that the superiors should to take personal interest to expose the employees to share their views by being friendly with them.

- The superiors have to create informal group meetings where people can share their experiences. Every day he has to review the progress and give suggestions whereby employees feel more comfortable and try to improve their performance.

- The superiors ought to appreciate the efforts of employees and help them wherever and whenever necessary. $\mathrm{He}$ has to create competition among the employees.

- The HR Managers have to involve concerned employees while preparing the HR Policies and practices in organizations like HPCL and CFL which leads to more involvement and commitment on the part of employees.

- From the analysis it was found that people do not trust each other, which is a bad atmosphere for any organization. The management has to take initiative to improve the confidence and trust in each other for better team work in the organization. To enable this culture, superiors have to be more informal and be friendly with the employees.

\section{CONCLUSION}

Human Resources Development is one of the most important activities of management. Organisational growth is not possible in the absence of trained manpower, the technological and global changes has necessitated the oragnisations to concentrate on qualitative human resources in the current developments' an organization success and failure is dependent on its human resources and their knowledge skills, abilities and more the adaptableness to the technological effectively linking people all the levels of management and process including decision making is the first and primary responsibility of the organization today. Organisations today have brought about considerable changes in the knowledge, skills, attitude and interpersonal behaviour. The concentration of the organizations should not only plan for developing human resources but also to impart a sense of responsibility in the employees.

\section{SCOPE FOR FURTHER RESEARCH}

In an attempt to address the limitations of this research, it is recommended that in order to achieve true value from the research, a number of organisations across industries should be selected to participate in the research. Utilising numerous organisations across industries will add value to explore the concept of a strong organisational culture.

\section{REFERENCES}

1. Wayne, F. Cascio (2003) : Managing Human Resources : Productivity Quality of work life, profits, Tata McGraw Hill, Delhi, p. 30.

2. Verma H.L., and McGarg (1997) : Human Resource Development : An Introduction Studies in Human Resource Development, Vol., Deep \& Deep, New Delhi, pp. 3

3. Becker B.S. Gerhart, B. (1996) : The impact of Human Resource Management on organizational performance: Progress and Prospects, Academy of Management, Journal, 39, pp. 779-801.

4. Rajiv Gandhi (1986) : Speech of Amethi, Times of India, 6th April 1986.

5. Yash Agarwal (1988): Education and Human Resource Development : Energy Challenges in the Regional context, common wealth publishers, New Delhi, p. 93.

6. Dayal, L. (1989) : HRD in Indian organizations : current perspectives and future, issues, Vikalps, Vol. 14(4) p. 22-25.

7. Yoshie Tomozumi Nakamura (2011) "The Role of Reflective Practices in Building Social Capital in Organizations from an HRD Perspective" Human Resource Development Review.

8. Moon K, Lee J, Oah S. The effects of safety leadership of manager and safety climate in the organization on the workers' safety behaviors. Journal of the Korean Society of Safety 2013; 28 (3): $\quad$ 66-72. Howell DC. Fundamental statistics for the behavioral sciences (8th ed.). Belmont (CA): Wadsworth CENGAGE Learning; 2014. p. 266.

9. Park KO. Organizational factors associated with safety and health managers' educational needs in Korean manufacturing industry. Korean Public Health Research 2016; 42 (1): 41-52. (in Korean) 
10. Planning Commission (1992) : Eighth Plan Volume-I, Government of India, p. 11.

11. Sheth N.R. (1993): What about Ethics and values in Management, Economic and Political Weekly, August 28, pp. M. 89-91.

12. Charles R. Greer (2001) : Strategic Human Resource Management: A general management approach, Pearson Education Pvt. Ltd., Delhi, p. 10.

13. Greasley, K., Bryman, A., Dairity, A., Price, A., Soetanto, R., \& King, N. (2005). Employee perceptions of empowerment. Employee relations, 27(4) 354-368.

14. Padmakali Mishra and Gopa Bhardwaj (2002) HRD climate an empirical among Private sector managers, India journal of industrial Relations p.66.

15. Dr. O.P. Mishra and Dr. D. Gangali, (2002) Current Trends in Extension Training, Indian Journal of Training and Development.

16. Hearn G, (1958). Theory Building in Social Work. Toronto, Canada: University of Toronto Press.

17. Lynham SA, (2000). "Theory Building in the Human Resource Development Profession". Human Resource Development Quarterly, Vol 11 No 2, pp 159-178.

18. Zboril-Benson, L., \& Magee, R. (2005). How quality improvement projects influence organisational culture. Healthcare Quartely, (no.8), ppl12. 